\title{
Gangliosides from Human Granulocytes: A Nano-ESI QTOF Mass Spectrometry Fucosylation Study of Low Abundance Species in Complex Mixtures
}

\author{
Wolfgang Metelmann, Jasna Peter-Katalinić \\ Medical Faculty, Institute for Medical Physics and Biophysics, University of Münster, Münster, Germany \\ Johannes Müthing \\ Technical Faculty, Institute for Cell Culture Technology, University of Bielefeld, Bielefeld, Germany
}

\begin{abstract}
Nano-ESI QTOF MS was used for sensitive mapping and sequencing of single molecular species in complex ganglioside mixtures obtained from human granulocytes, where the fucosylated carbohydrate chains of granulocyte gangliosides carry sLe ${ }^{x}$ and VIM-2 epitopes postulated to interact with E-selectin of the blood vessel wall in the early phase of the inflammation process. Functionally relevant components are expressed only at a low level, but using the negative ion detection it is possible to trace and identify such species, which were not detectable even by TLC. Advantage of the low-energy CID fragmentation for low abundance components of the complex ganglioside mixtures in the negative ion mode is to produce clear-cut series of fragment ions for sequencing. Fucosylation analysis carried out for each molecular species by MS/MS permits the clear distinction between sLe ${ }^{x}$ and VIM-2 epitope. VIM-2 epitope was expressed in all species with a longer sugar core, while in the short oligosaccharide chain with a sLe ${ }^{\mathrm{x}}$ only, using biological material at a mid-femtomol level detection. (J Am Soc Mass Spectrom 2001, 12, 964-973) (c) 2001 American Society for Mass Spectrometry
\end{abstract}

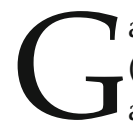
angliosides, sialylated glycosphingolipids (GSLs), are amphiphatic molecules consisting of a hydrophilic oligosaccharide chain and a hydrophobic lipid anchor. To a long chain aminodiol, like sphingosine (4-sphingenine, d18:1) as a lipid backbone, fatty acids of different lengths are attached to the amino group, forming the ceramide moiety. Gangliosides are postulated to play important roles in modulation of function of membrane-associated proteins and in cellcell recognition phenomena. E-selectin, a carbohydratebinding cell adhesion molecule [1], is induced by cytokines such as TNF- $\alpha$ or IL- $1 \beta$ on the surface of endothelial cells. It mediates the docking of granulocytes to the blood vessel wall and their rolling along the endothelial cell surface to the loci of inflammation [2]. A few lines of evidence strongly suggest that certain fucosylated monosialogangliosides with linear poly-N-

Published online May 30, 2001

Address reprint requests to Dr. J. Peter-Katalinić, Laboratory of Biomedical Analysis, Institute for Medical Physics and Biophysics, University of Münster, Robert-Koch Str. 31, D-48149 Münster, Germany. E-mail: jkp@unimuenster.de

This paper was presented at the 48th ASMS Conference on Mass Spectrometry and Allied Topics, June 2000, Long Beach, CA, USA. acetyllactosaminyl chains in the plasma membrane of granulocytes are capable of binding to E-selectin [3-5].

To enhance studies on biological functions of gangliosides [6], main improvements in their purification [7] and structural analysis [8,9] have been made. As recently reported, the hybrid quadrupole time-of-flight (QTOF) mass spectrometer $[10,11]$ is a powerful tool for analysis of complex ganglioside mixtures. In a previous work, sialylated neolactotetraosyl- and neolactohexaosylceramides (IV ${ }^{3} \mathrm{Neu} 5 \mathrm{Ac}-\mathrm{nLc} 4 \mathrm{Cer}$ and $\mathrm{VI}^{3} \mathrm{Neu}$ $5 \mathrm{Ac}^{-n L c_{6} \mathrm{Cer}}$, respectively) from human granulocytes were used as models for fragmentation studies by nano-electrospray ionization (ESI) tandem mass spectrometry (MS/MS) on a QTOF analyzer. Particular attention was paid toward determination of the sialic acid moiety attachment site in the type $2 \mathrm{~N}$-acetyllactosamine sugar chain [12]. The aim of the present study is to explore potentials and limits of nano ESI-QTOF analysis for detection and full characterization of such components, which are present in complex ganglioside mixtures in low amounts, but may play essential roles in biologically significant interactions. Such gangliosides are the minor components of the described human granulocyte ganglioside fraction, which contains exclusively terminally $\alpha 2,3$-sialylated monosialogangliosides 
[13]. Determination of fucose attachment sites at penultimate, inner core, or proximal GlcNAc moieties in the poly-N-acetyllactosaminyl chains in the mass range of $\mathrm{m} / \mathrm{z} 1600$ to 3000 was one of the main objectives.

\section{Experimental}

\section{Gangliosides from Human Granulocytes}

Human leukocytes enriched with granulocytes were prepared from buffy coats and gangliosides were isolated and purified as previously described [5, 14]. Chloroform and methanol of analytical grade (Merck, Darmstadt, Germany) were distilled before use. Briefly, GSLs were extracted with chloroform/methanol (2/1) and $(1 / 2)$, each by volume. The combined extracts were evaporated and partitioned according to Folch et al. [15]. Gangliosides of Folch upper phases were separated from neutral GSLs by anion exchange chromatography on diethylaminoethyl (DEAE)-Sepharose CL-6B (Pharmacia Fine Chemicals, Freiburg, Germany) as reported by Müthing et al. [16]. Total gangliosides were eluted with $0.45 \mathrm{M}$ ammonium acetate in methanol. After evaporation and desalting by dialysis, the ganglioside fraction was incubated for $1 \mathrm{~h}$ at $37^{\circ} \mathrm{C}$ in $1 \mathrm{~N}$ $\mathrm{NaOH}$ to hydrolyze phospholipids, followed by neutralization with acetic acid and dialysis. Gangliosides were then further purified by Iatrobeads 6RS-8060 chromatography (Macherey-Nagel, Düren, Germany) as described by Ueno et al. [17]. Stepwise elution was performed with chloroform/methanol (85/15), (3/1), (2/ $1),(1 / 2)$, each by volume, and finally methanol. A neolacto-series ganglioside fraction lacking GM3 species was obtained in the chloroform/methanol $(1 / 2)$ eluate and was used for preparative high performance liquid chromatography (HPLC) analysis of gangliosides as explained in the following section.

\section{High Performance Liquid Chromatography}

The separation of $\alpha 2-3$ - and $\alpha 2-6$-sialylated monosialogangliosides HPLC was carried out on the Superformance universal glass cartridge device (Merck, Darmstadt) published earlier in detail [5, 13]. A glass cartridge $(150 \mathrm{~mm} \times 10 \mathrm{~mm})$ filled with Fractogel EMD TMAE-650(S) (Merck) was loaded with the GM3 depleted ganglioside fraction (chloroform/methanol (1/2) eluate of the Iatrobeads chromatography, see above). TMAE-Fractogel bound gangliosides were eluted with a linear ammonium acetate gradient, pooled, and desalted. The pool of ganglioside fractions 16 to 21, comprising terminally $\alpha 2-3$ sialylated neolacto-type monosialogangliosides, was used for nano-ESI-QTOF MS/MS analysis in this study.

\section{Thin-Layer Chromatography}

Gangliosides were separated on Silica Gel 60 precoated high-performance thin-layer chromatography plates
(HPTLC-plates, size $10 \mathrm{~cm} \times 10 \mathrm{~cm}$, thickness $0.2 \mathrm{~mm}$, Merck) for $25 \mathrm{~min}$ using a mixture of chloroform/ methanol/water (24/17/4, each by vol.) supplemented with $2 \mathrm{mM} \mathrm{CaCl}_{2}$, and visualized with orcinol [18].

\section{Sample Preparation}

The sample containing the complex ganglioside mixture was dissolved in pure methanol (SupraSolv grade for organic trace analysis, Merck, Darmstadt, Germany) to a concentration of approximately $0.03 \mathrm{~g} / 1$ and sprayed under optimized conditions with flow rates of approximately $20 \mathrm{nl} / \mathrm{min}$. Acquisition time for the MS spectrum was $2 \mathrm{~min}$ and 15 to $45 \mathrm{~min}$ for acquisition of the MS/MS spectra. Additionally, comparing experiments with a bovine brain ganglioside mixture, Cronassial (Fidia Research Labs, Abano Terme, Italy) were performed, containing 21\% GM1a, 40\% GD1a, 16\% GD1b, and 19\% GT1b, and dissolved in SupraSolv methanol to a concentration of $0.001 \mathrm{~g} / 1$ for MS/MS analysis and $10^{-4} \mathrm{~g} / 1$ for MS mapping.

\section{Mass Spectrometry}

The capillaries used for electrospray have been made in-house from borosilicate glass (Hilgenberg, Malsfeld, Germany) using a pipette puller (Model 720, David Kopf Instruments, Tujunga, CA, USA). The high voltage is applied via a steel wire to the sample solution as capillaries are used without further metal coating. Capillary voltages for all experiments were between $1.1 \mathrm{kV}$ and $1.2 \mathrm{kV}$. Negative ion mode was used exclusively for the ganglioside analysis. All experiments were obtained with a QTOF Mass Spectrometer equipped with a nanospray manipulator (Micromass, Manchester, UK). To detect all molecular species as singly charged ions and to reduce the amount of doubly- and triply charged molecular ions (to simplify spectra interpretation), the voltage on the counter electrode was set to $150 \mathrm{~V}$ [12]. The voltage at the second orifice leading to the next pressure stage was set to $4 \mathrm{~V}$, contributing to a significant stabilization of the total ion current and therefore leading to a significantly higher ionization yield. Resolution of investigated signals was measured as full width at half maximum (FWHM) and was about 5500, slightly decreasing at the higher mass signals. After selecting the precursor ion of interest with the first quadrupole by increasing the DC/AC ratio, collisional activation was applied to obtain fragment ions for MS/MS sequencing. The pressure in the collision cell was approximately $3.8 \cdot 10^{-5}$ mbar using argon as a collision gas. The collision energy was set to values, reducing the intensity of the precursor ion signal to one third of its original intensity to obtain high fragment ion abundance within a short period of time. Ions are guided in a radiofrequency (RF)-only hexapole and orthogonally extracted to a grid reflector. In all experiments the orthogonal TOF analyzer with time to digital conversion of the signal detected [19] combined with a 


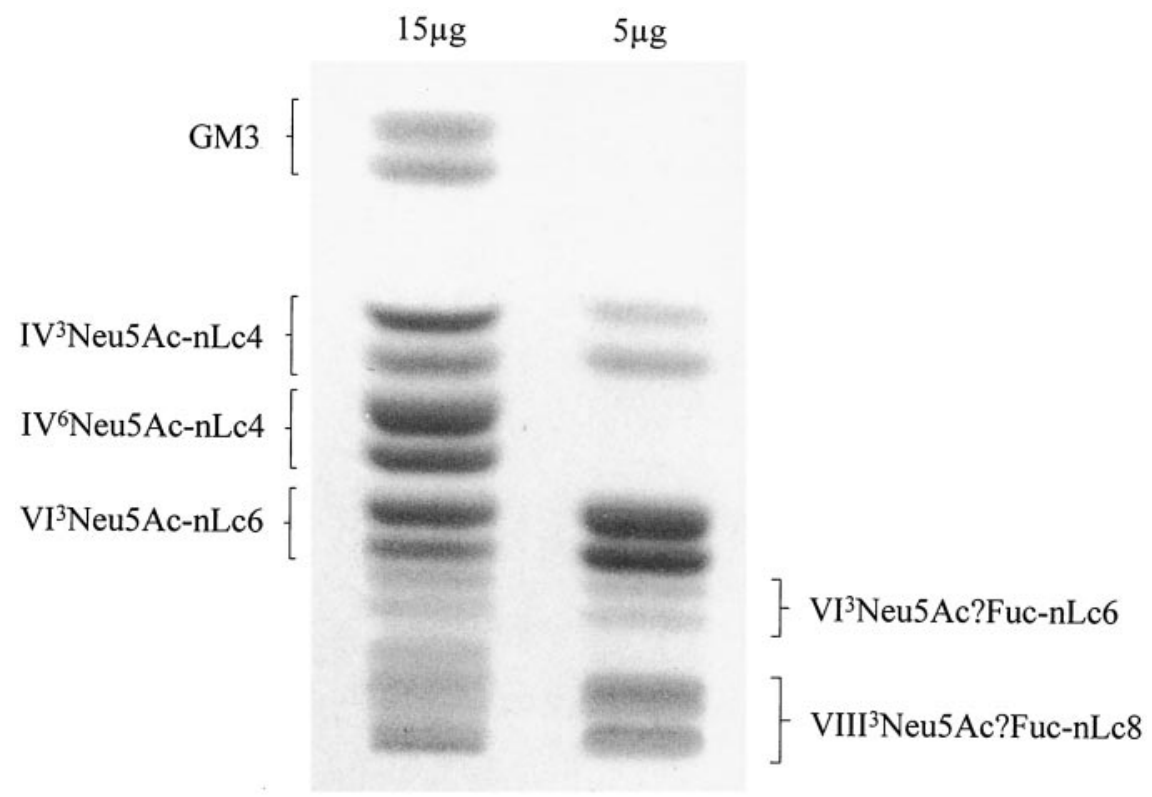

a)

b)

Figure 1. Orcinol stained thin-layer chromatogram of gangliosides from human granulocytes. Lane a: $15 \mu \mathrm{g}$ of total gangliosides; lane b: $5 \mu \mathrm{g}$ of $\alpha 2,3$-sialylated monosialogangliosides, obtained by TMAE-Fractogel HPLC, used for structural analysis in this study.

quadrupole ion guide (hybrid quadrupole-TOF instrument) leads to significant improvement of sensitivity and resolution. The duty cycle of this instrument, quoted as $20-22 \%$ [20], depends on factors like ratio between lens opening for orthogonal extraction and the size of the multichannel plate (MCP) detector, or the velocity of ions moving into the extraction area between two voltage pulses.

In general, the nomenclature from Domon and Costello [21] is used for assignment of fragment ions. To embrace the observed charge state of fragment ions, e.g., $\mathrm{Y}_{8 / 3}$ ion in the MS/MS spectrum shown in Figure $3 a$, we applied an (also correct) description, $\mathrm{Y}_{8} / \mathrm{Y}_{3}$. The designation of glycosphingolipids follows the IUPACIUB recommendations [22, 23].

\section{Results and Discussion}

The orcinol stain of total gangliosides TLC separation and $\alpha 2,3$-sialylated monosialogangliosides from human granulocytes are shown in Figure 1 (lane a and b, respectively). Human granulocyte gangliosides, e.g., $\mathrm{IV}^{3} \mathrm{Neu} 5 \mathrm{Ac}-\mathrm{nLc} 4 \mathrm{Cer}$ or $\mathrm{IV}^{6} \mathrm{Neu} 5 \mathrm{Ac}-\mathrm{nLc4Cer}$, migrate differently, giving rise to double bands due to the substitution of the sphingosine (d18:1) with C24:1 (upper band) and C16:0 fatty acid (lower band) [5, 14]. In this fraction, $\alpha 2,3$-sialylated monosialogangliosides $\mathrm{IV}^{3} \mathrm{Neu} 5 \mathrm{Ac}-\mathrm{nLc4Cer}$ and $\mathrm{VI}^{3} \mathrm{Neu} 5 \mathrm{Ac}-\mathrm{nLc6Cer}$ (Figure 1 , lane b), already structurally characterized, are present [12]. Minor components of this fraction, which also migrates as double bands, could possibly be assigned to fucosylated gangliosides. Deduced from migration be- haviour in the TLC runs, these low abundance species could belong to the nLc6Cer or nLc8Cer species with unknown number and position(s) of fucose moieties. This mixture was submitted to the nano-ESI QTOF MS analysis in the negative ion mode, where a ganglioside map (Figure 2) of this complex mixture was obtained. Besides the already characterized major components the low abundance ions were present (marked with an asterisk). These ions were chosen as precursor ions for sequencing by MS/MS to investigate if the fragmentation under low-energy collision-induced dissociation (CID) conditions can provide the evidence about fucosylation status and discern patterns for assignment of the fucose attachment to the neolactosyl core. The presence of two biologically relevant epitopes, differing in the position of the fucose in the neolacto-type oligosaccharide chain (sialyl-Lewis ${ }^{x}\left(\mathrm{sLe}^{\mathrm{x}}\right)$ and VIM-2) (Figure $4 \mathrm{~b}$ and Figure $5 \mathrm{~b}$ ) have been indicated to be present in the sample by TLC immunostaining.

The precursor ion at $m / z 2246.9$ was chosen for MS/MS sequencing to verify the absence of fucose in this species. The spectrum (Figure 3a) mainly consists of $\mathrm{Y}$ ions like the $\mathrm{Y}_{8}$ at $\mathrm{m} / \mathrm{z} 1955.9$ (loss of sialic acid), $\mathrm{Y}_{7}$ at $\mathrm{m} / \mathrm{z} 1793.9$ (loss of outer galactose) and $\mathrm{Y}_{6}$ at $\mathrm{m} / \mathrm{z} 1590.8$ (loss of outer $\mathrm{N}$-acetylglucosamine). The complete sequence of the nLc8Cer oligosaccharide chain without fucose is clearly discerned. All fragments containing a terminal $\mathrm{N}$-acetylglucosamine $\left(\mathrm{Y}_{7}, \mathrm{Y}_{5}, \mathrm{Y}_{3}\right)$ are accompanied by respective $Z$-ions after neutral loss of water. Additionally, some $B$ ions like $B_{3}$ at $m / z 655.2$ or $B_{4}$ at $\mathrm{m} / \mathrm{z} 817.3$ are detected at low abundance. Internal fragment ions like $\mathrm{Y}_{8} / \mathrm{B}_{6}$ at $m / z 1256.4, \mathrm{Y}_{6} / \mathrm{B}_{6}$ at $m / z 891.3$ or 


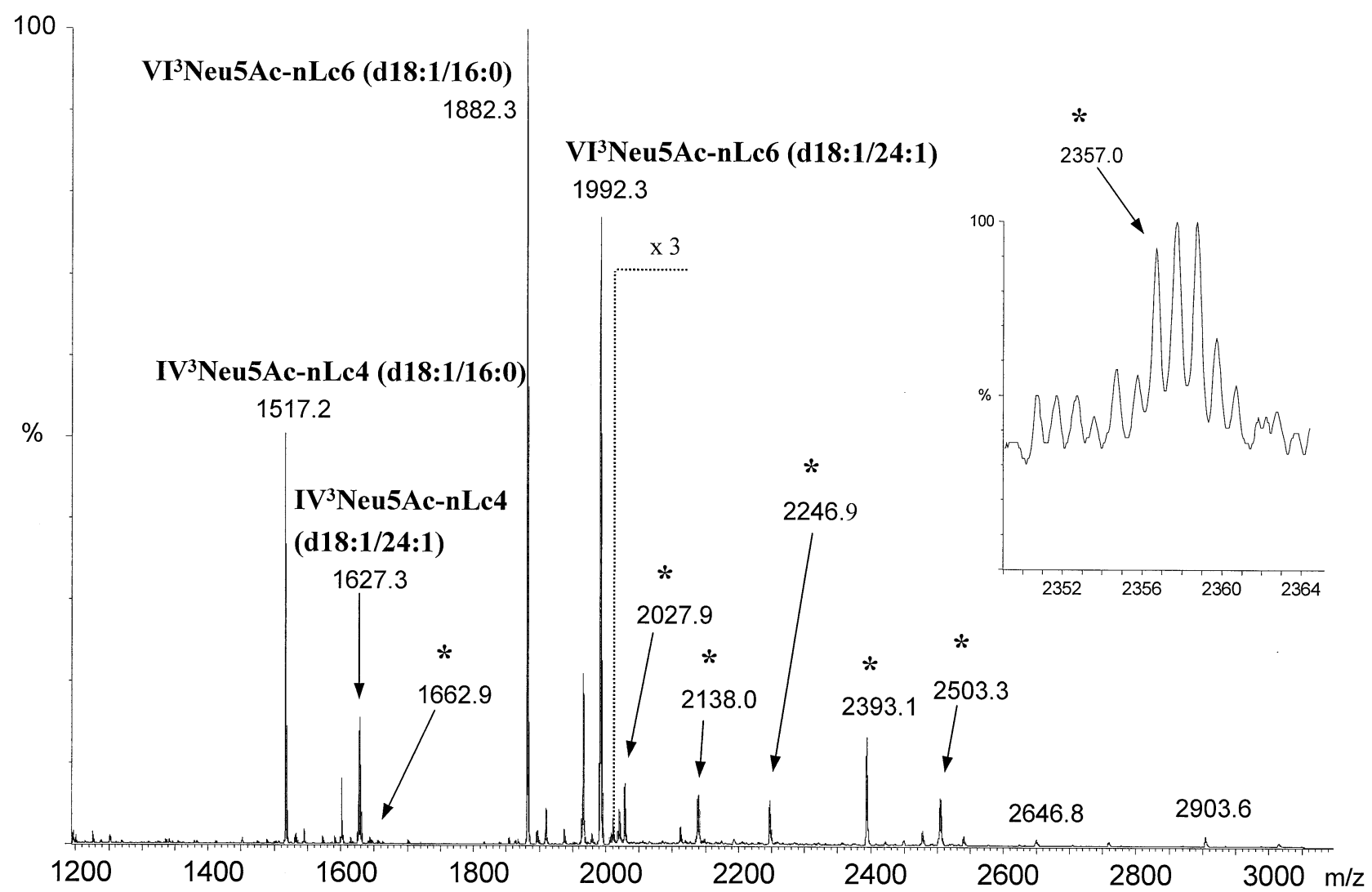

Figure 2. MS spectrum of the investigated ganglioside mixture. Only the region of molecular ions is presented. Signals marked with an asterisk were investigated in detail.

$\mathrm{Y}_{6} / \mathrm{B}_{7}$ at $m / z 729.3$ provide additional evidence for the core chain with alternating Gal and GlcNAc units, a terminal neuraminic acid and a d18:1/16:0-ceramide (Figure 3b), confirming the TLC immunostaining results. The nLc8Cer molecular ion with a C24:1 fatty acid in the ceramide part at $\mathrm{m} / \mathrm{z} 2357.0$ is barely detectable in the mapping spectrum (Figure 2, zoomed part). Although it was possible to carry out MS/MS analysis (spectrum not shown) and the product ions were obtained at much lower $\mathrm{s} / \mathrm{n}$ ratio than for the d18:1/16:0 species: only the high abundance signals, such as the $\mathrm{Y}_{4}$ ion at $\mathrm{m} / \mathrm{z} 1335.8$ or the $\mathrm{Y}_{8}$ at $\mathrm{m} / \mathrm{z} 2065.9$ (loss of sialic acid), gave evidence of the proposed structure.

In the MS/MS spectrum of the low abundance precursor ion at $m / z 1662.9$ (Figure 4a), Y-type ions like the $\mathrm{Y}_{3 \beta}$ ion at $m / z 1516.9$ (loss of fucose) provide a direct hint for its fucosylation status via a relationship to the non-fucosylated structure of the molecular species at $m / z$ 1517.0 (IV ${ }^{3}$ Neu5Ac-nLc4) in Figure 2. The $\mathrm{Y}_{4 \alpha}$ ion at $\mathrm{m} / \mathrm{z} 1371.8$ (loss of sialic acid) or the abundant $\mathrm{Y}_{2}$ ion at $m / z 860.7$ are produced as well as a number of internal fragments (e.g., $\mathrm{Y}_{4 \alpha} / \mathrm{B}_{3}$ at $m / z 510.2, \mathrm{Y}_{3 \beta} / \mathrm{B}_{3}$ at $m / z$ 655.2, or $\mathrm{Y}_{3 \alpha} / \mathrm{Y}_{3 \beta}$ at $m / z$ 1063.7) supporting this relationship. Besides, the ion at $m / z 680.6$ corresponding formally to the $Z_{1}$ ion, rarely detected in the native ganglioside low-energy CID MS/MS, was present. The $\mathrm{Y}_{3 \alpha}$ ion at $m / z$ 1209.8, presented in the frame, is of diagnostic relevance for determination of the fucose attachment site. Sialic acid and one galactose are cleaved off but the fucose is still present, suggesting attachment to the $\mathrm{N}$-acetylglucosamine which would be in agreement with the immunostaining results. The corresponding characteristic tetrasaccharide $\mathrm{B}_{3}$ ion (sequence: Neu5AcGal-GlcNAc) was not detected, but deduced from the number of $\mathrm{N}$-acetylglucosamine residues in the oligosaccharide chain, the epitope structure of sLe ${ }^{x}$ (Figure $4 b)$ is the single feasible structure.

In the higher mass range, the ion at $\mathrm{m} / \mathrm{z} 2138.0$ was selected for fragmentation related to fucosylation status (Figure 5a). In its MS/MS spectrum a complete series of $\mathrm{Y}$ ions was detected starting with the $\mathrm{Y}_{3 \beta}$ at $\mathrm{m} / \mathrm{z} 1991.9$ (loss of fucose) characteristic for the core of the sialylated nLc6-(d18:1/24:1) structure. The $\mathrm{Y}_{6 \alpha}$ ion at $\mathrm{m} / \mathrm{z}$ 1846.9 (loss of sialic acid) and the cleavage at each of the following sugar units is observed: $\mathrm{Y}_{5 \alpha}$ at $m / z 1684.8, \mathrm{Y}_{4 \alpha}$ at $m / z$ 1481.8. The ceramide ion $Y_{0}$ at $m / z 646.6$ is indicative for the presence of a C24:1 fatty acid linked to the sphingoid base. In this MS/MS spectrum both characteristic ions for the attachment of the fucose moiety were found: $Y_{3 \alpha}$ ion at $m / z 1319.8$ and the corresponding $\mathrm{B}_{5}$ ion at $\mathrm{m} / \mathrm{z} 1166.4$, providing evidence for the VIM-2 structural epitope (Figure 5b). The high abundance signal at $m / z 1335.8\left(\mathrm{Y}_{4 \alpha} / \mathrm{Y}_{3 \beta}\right)$ could arise 


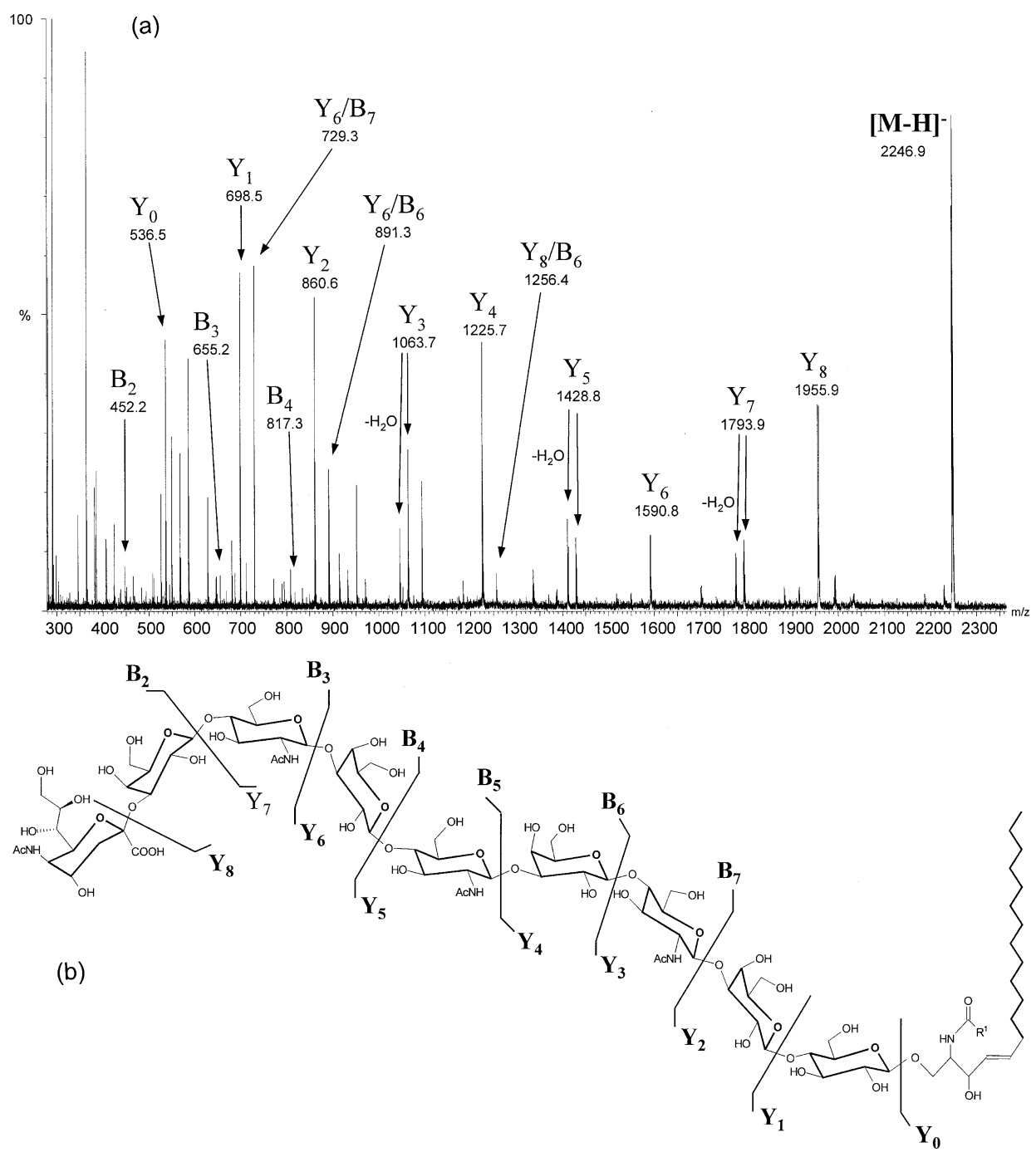

Figure 3. (a) MS/MS spectrum of the ion at $m / z 2246.9$ as a precursor. All fragment ions appear as singly charged species. (b) Proposed molecular structure of the molecular species characterized by the ion at $m / z 2246.9$. $R$ is a fatty acid residue with either a C16:0 or a C24:1 chain.

from the loss of a sLe ${ }^{\mathrm{x}}$ structural epitope at the nonreducing terminus of the carbohydrate chain as well as from a loss of Neu5Ac-Gal-GlcNAc trisaccharide besides an additional, unspecific loss of fucose from another position in the chain. The latter assumption is supported by the presence of fucosylated fragment ions at lower $\mathrm{m} / \mathrm{z}$ than the observed $\mathrm{Y}_{4 \alpha} / \mathrm{Y}_{3 \beta}$ and by the corresponding $\mathrm{B}_{3 \alpha}$ ion at $\mathrm{m} / \mathrm{z} 655.2$ without fucose. A conclusion based on this evidence is supportive for the VIM-2 epitope structure at the nonreducing end.

The structural relationship of the ions at $\mathrm{m} / \mathrm{z} 2138.0$ and $m / z 2027.9$ (data not shown) is clearly deducible from values of their respective molecular masses and Y-fragment ions, differing by $110.1 \mathrm{Da}$ from those shown in Figure 5a. This feature is typical for components with identical carbohydrate chain but different fatty acids in the ceramide part (see also Figure 1). Due to the presence of two $Y$ ions: $Y_{4 \alpha} / Y_{3 \beta}$ at $m / z 1225.7$ and $\mathrm{Y}_{3 \alpha}$ at $m / z$ 1209.7, a distinction between sLe ${ }^{\mathrm{x}}$ and VIM-2 structure would be difficult. Using the evidence from the corresponding B-ions, the distinction between these two epitopes is, however, possible, deduced from the presence of the VIM-2 characteristic $B_{5}$ ion at $m / z 1166.4$ at low abundance and from the absence of the fucosylated $\mathrm{B}_{3 \alpha}$ ion characteristic for $\mathrm{sLe}$.

The series of $Y$ fragment ions in the MS/MS spectrum of the precursor ion at $\mathrm{m} / \mathrm{z} 2503.3$ (Figure 6a) diagnostic for the sialylated nLc8 family, was accompanied by the $Y_{0}$ ion at $m / z$ 646.6, commonly observed for a (d18:1/24:1) ceramide. After loss of sialic acid resulting in $Y_{8 \alpha}$ ion at $m / z 2212.6$ an alternating stepwise loss of galactose and $\mathrm{N}$-acetylglucosamine was documented by $\mathrm{Y}_{7 \alpha}$ ion at $m / z 2050.5, \mathrm{Y}_{6 \alpha}$ ion at $m / z 1847.2, \mathrm{Y}_{5 \alpha}$ ion at $\mathrm{m} / \mathrm{z} 1685.1, \mathrm{Y}_{4}$ ion at $\mathrm{m} / \mathrm{z} 1336.0, \mathrm{Y}_{3}$ ion at $\mathrm{m} / \mathrm{z} 1173.9, \mathrm{Y}_{2}$ ion at $m / z$ 970.7. The $Y_{5 \alpha}$ ion (framed) at $m / z 1685.1$ and the $\boldsymbol{B}_{5}$ ion at $m / z 1166.4$ (framed, zoomed part in Figure 6a) support the evidence for the VIM-2 structural epitope (Figure $6 \mathrm{~b}$ ). It should be mentioned that the 


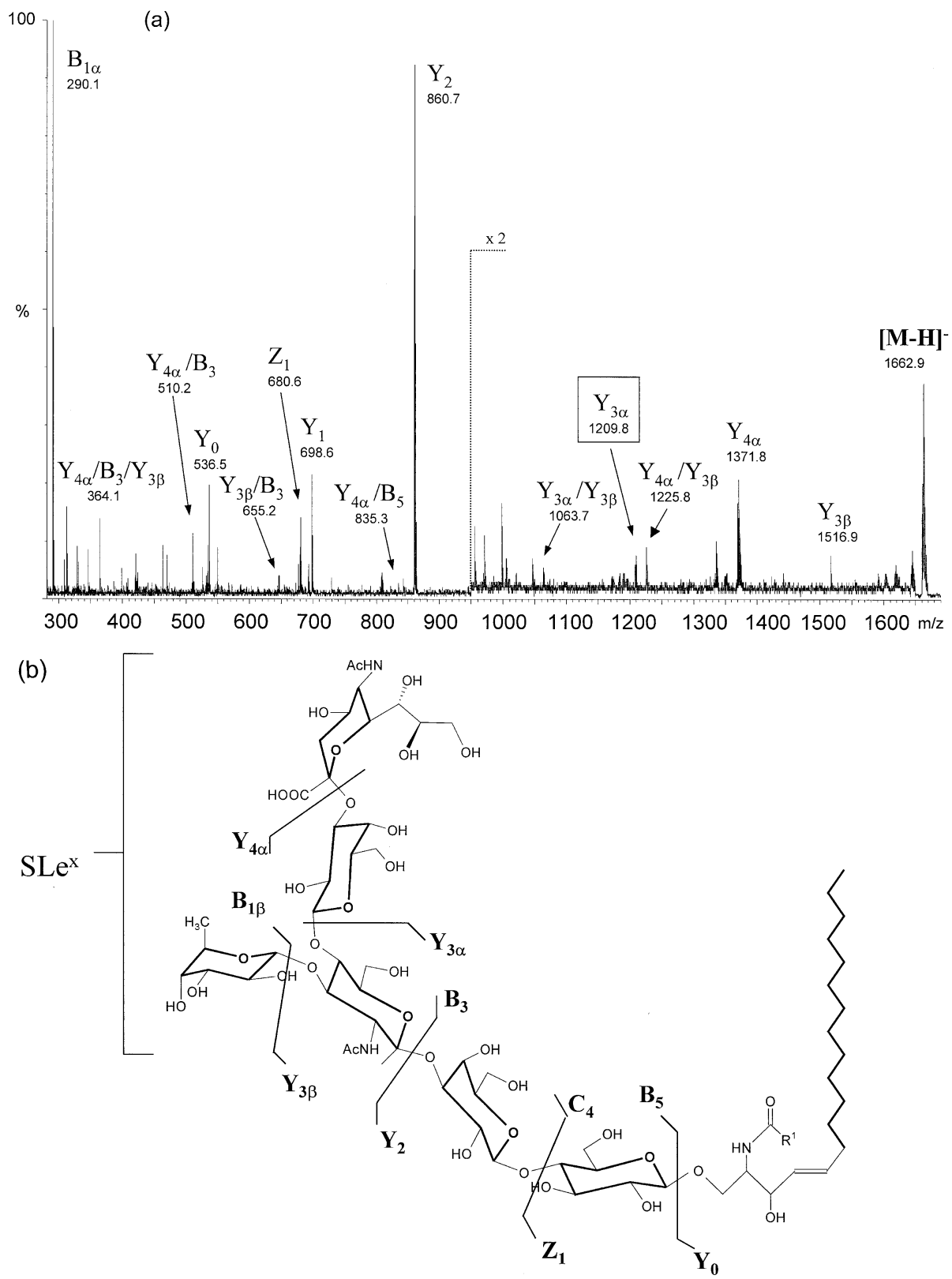

Figure 4. (a) MS/MS spectrum of the molecular ion at $m / z 1662.9$ as a precursor. All fragment ions appear as singly charged species. (b) Proposed molecular structure of the molecular species characterized by the ion at $\mathrm{m} / \mathrm{z} 1662.9$.

$\mathrm{Y}_{6 \alpha} / \mathrm{Y}_{5 \beta}$ ion at $\mathrm{m} / \mathrm{z} 1701.2$ could arise either from a loss of a sLe ${ }^{\mathrm{x}}$ epitope or from a loss of Neu5Ac-Gal-GlcNAc besides an additional, unspecific loss of fucose from another position in the chain. A sLe ${ }^{\mathrm{x}}$ characteristic $\mathrm{B}_{3 \alpha}$ ion (calculated $m / z$ 801.3) was not detected, contrary to the nonfucosylated $B_{3 \alpha}$ at $m / z$ 655.3.

The MS/MS spectrum of the ion at $m / z 2393.1$ (not shown), reveals the structural relationship of the molecular ions at $\mathrm{m} / \mathrm{z} 2503.3$ and $\mathrm{m} / \mathrm{z}$ 2393.1. In the comparison of the $\mathrm{Y}$-ion series, mass differences depict the same structural homology as mentioned before with the ion pair at $m / z 2138.0$ and $m / z$ 2027.9, where each observed fragment ion has its counterpart in the spectrum shown in Figure 6a but differs by 110.1 Da. A number of homologous inner fragments like $\mathrm{Y}_{6 \alpha} / \mathrm{B}_{7}$ at $m / z$ 875.4, $\mathrm{Y}_{5 \alpha} / \mathrm{B}_{9}$ at $m / z 1037.5$, or $\mathrm{Y}_{6 \alpha} / \mathrm{B}_{7} / \mathrm{Y}_{5 \beta}$ at $\mathrm{m} / \mathrm{z} 729.3$ support this fact. In the carbohydrate moiety, common for both precursor ions, particular attention was directed toward two $\mathrm{Y}$ ions, $\mathrm{Y}_{6 \alpha} / \mathrm{Y}_{5 \beta}$ at $m / z \quad 1590.9$ and $Y_{5 \alpha}$ at $m / z$ 1574.9, present in the spectra. Because the fucosylated $B_{3 \alpha}$ ion that would have been characteristic for $\mathrm{sLe}^{\mathrm{x}}$ was not detected, and the $\mathrm{B}_{5}$ ion was observed at $m / z 1166.4$ (VIM-2) with only low abundance, the distinction between sLe $^{\mathrm{x}}$ and VIM-2 structures could not confidently be made. 

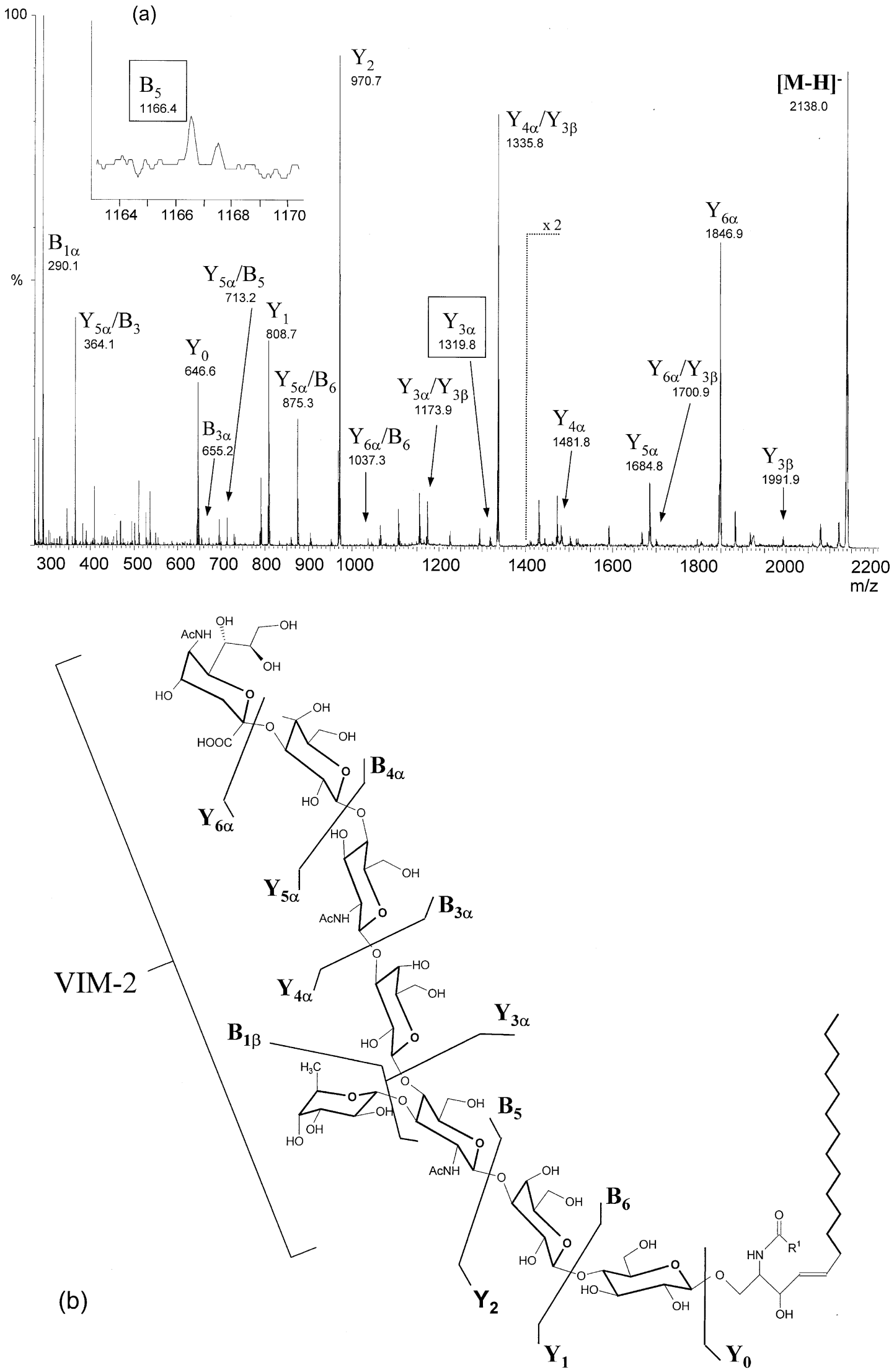

Figure 5. (a) MS/MS spectrum of the molecular ion at $m / z 2138.0$ as a precursor. All fragment ions appear as singly charged species. (b) Proposed molecular structure of molecular species characterized by ions at $\mathrm{m} / \mathrm{z} 2027.9$ and $\mathrm{m} / \mathrm{z} 2138.0$. $R$ is a fatty acid residue with either a C16:0 or a C24:1 chain. 

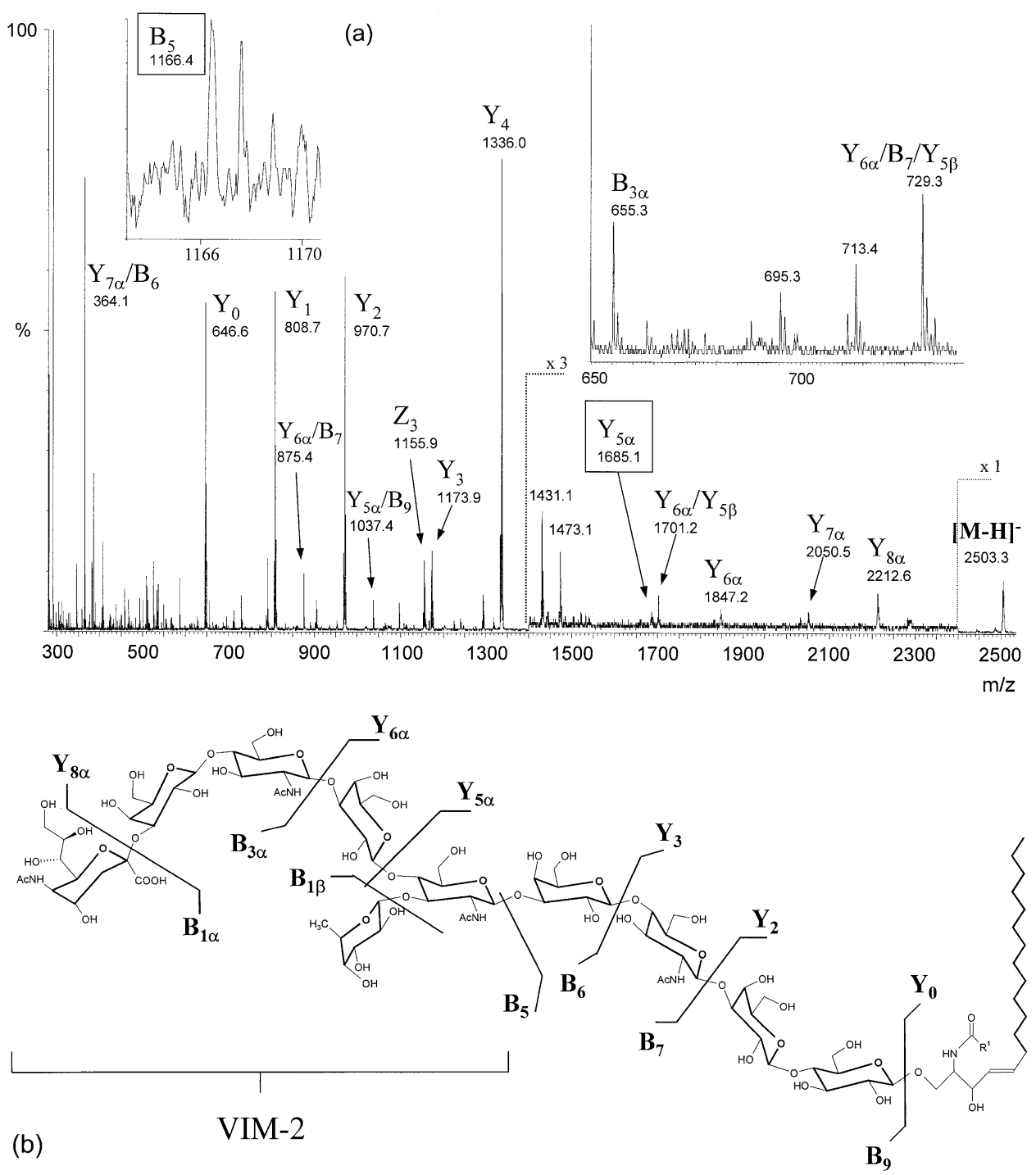

Figure 6. (a) MS/MS spectrum of the molecular ion at $m / z 2503.3$ as a precursor. All fragment ions appear as singly charged species. (b) Proposed molecular structure of molecular species characterized by ions at $m / z 2393.1$ and $m / z 2503.3$. $R$ is a fatty acid residue with either a C16:0 or a C24:1 chain.

\section{Sample Consumption and Detection Limit}

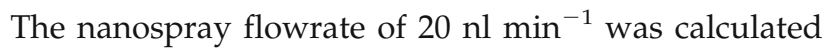
in experiments using methanol as a solvent and the laboratory made capillaries, from which it was possible to detect ions for at least one hour. The overall consumption for the particular experiment was estimated after measuring the volume of the retained liquid, where the values obtained had a deviation of about $\pm 50 \%$, because of the scattering quality of the spray capillaries, as was observed by others also [24].

For a short MS, 2 min acquisition time and $40 \mathrm{nl}$ of the ganglioside mixture were required. The overall sample consumption for MS/MS was calculated to be in a range between $3 \mathrm{pmol}$ (ion at $\mathrm{m} / \mathrm{z} 2138.0$ ) and $16 \mathrm{pmol}$ (ion at $\mathrm{m} / \mathrm{z}$ 1662.9), depending on different acquisition times (15 to $45 \mathrm{~min}$ ). These relatively high amounts are a concession to the importance of low abundance sig- nals in the product ion spectra which would allow distinction between different biological epitopes and would probably not be detectable at lower sample concentration.

To demonstrate that the QTOF sensitivity for glycosphingolipids can reach even a femtomol or subfemtomol range, a MS spectrum was acquired from the Cronassial standard ganglioside mixture, where a clear detection of each predicted ganglioside species and their respective ions $[\mathrm{M}-\mathrm{H}]^{-}$could be achieved after $1.25 \mathrm{~min}$ acquisition time $(25 \mathrm{nl})$. The sample consumption for a single molecular ganglioside species in MS should be considered as a relative amount of each molecular species. For GM1a species it was calculated to be $340 \mathrm{amol}$, for GD1a and GD1b species $751 \mathrm{amol}$, and for GT1b species 215 amol. Clear fragmentation spectra by MS/MS with a s/n ratio 4:1 even for low 
Table 1. Compounds investigated in this study

\begin{tabular}{|c|c|c|c|c|c|}
\hline $\begin{array}{l}\text { Observed } \\
\mathrm{m} / \mathrm{z}\end{array}$ & Core structure & Fucosylated & Epitope & Name (IUPAC) & Spectrum shown in \\
\hline 1662.9 & $\mathrm{nLc4}$ & mono & $s L e^{x}$ & IV Neu5AcIlIFucnLc4 (d18:1/16:0) & Figure $4 a$ \\
\hline 2027.9 & $\mathrm{nLc6}$ & mono & VIM- $2^{a}$ & VI ${ }^{6}$ Neu5AcIlIFucnLc6 (d18:1/16:0) & not shown \\
\hline 2138.0 & $\mathrm{nLc6}$ & mono & VIM-2 & $\mathrm{VI}^{6} \mathrm{Neu} 5 \mathrm{AcIlIFucnLc6}$ (d18:1/24:1) & Figure $5 a$ \\
\hline 2246.9 & $\mathrm{nLc8}$ & non & - & VIII ${ }^{8}$ Neu5AcnLc8(d18:1/16:0) & Figure $3 a$ \\
\hline 2357.1 & $\mathrm{nLc8}$ & non & - & VIII ${ }^{8}$ eu5AcnLc8(d18:1/24:1) & not shown \\
\hline 2393.1 & $\mathrm{nLc8}$ & mono & VIM- $2^{a}$ & VIII ${ }^{8}$ eu5AcVFucnLc8 (d18:1/16:0) & not shown \\
\hline 2503.3 & $\mathrm{nLc8}$ & mono & VIM-2 & VIII ${ }^{8}$ eu5AcVFucnLc8 (d18:1/24:1) & Figure $6 a$ \\
\hline
\end{tabular}

${ }^{\mathrm{a}}$ Only a diagnostic $\mathrm{Y}$ ion was detected.

abundance signals were obtained within $2 \mathrm{~min}$ for GM1a and 50 min acquisition time for GT1b, which makes a consumption of $0.55 \mathrm{fmol}$ of GM1a and $8.6 \mathrm{fmol}$ of GT1b. These values, however, theoretically include the natural distribution of different fatty acids attached to the sphingosine so that the real amount of material for one isolated molecular ion is even lower.

It is important to mention that the relative abundance of single ion species in the MS spectrum of the Cronassial mixture cannot be directly correlated with values for the relative amount of the gangliosides obtained probably from densitometrical measurements from TLC plates: although GM1a is only contained with $21 \%$, it shows the most abundance signals in the negative ion mode spectrum (approximately $82 \%$ ).

\section{Conclusions}

Using ESI MS and low-energy CID MS/MS a complex ganglioside mixture was analyzed on the QTOF instrument to trace and identify components, which may play a significant role in cell adhesion. It was possible to demonstrate the feasibility of detection and sequencing of gangliosides, which are present at low abundance, using negative ion detection mode. The position of the fucose attachment was determined for each molecular species. MS/MS spectra interpretation permits the clear distinction between sLe ${ }^{x}$ and VIM-2 epitope structures even if only low abundance fragment ions are present, due to the high dynamic range of the instrument. It can be postulated that the VIM-2 epitope is expressed in all species with a longer sugar core, as in nLc6 and nLc8, while in the short oligosaccharide chain of nLc4 whithout the possibility of VIM-2 formation a sLe epitope only is expressed. Seven low abundance molecular species, investigated and structurally characterized in this study are listed in Table 1. Using this approach a fucosylated IV ${ }^{3}$ Neu5Ac-nLc4 species not detected in the TLC chromatogram (Figure 1) was structurally characterized demonstrating the sensitivity of this experiment. Furthermore, two nonfucosylated species of $\mathrm{VIII}^{3} \mathrm{Neu} 5 \mathrm{Ac}-$ nLc8 with molecular ions at $\mathrm{m} / \mathrm{z} 2246.9$ and $\mathrm{m} / \mathrm{z} 2357.0$ (Figure 2) were identified. The MS/MS data supported the original hypothesis that the TLC bands can be assigned to the fucosylated $\mathrm{VI}^{3} \mathrm{Neu} 5 \mathrm{Ac}-\mathrm{nLc6}-\mathrm{Cer}$ and $\mathrm{VIII}^{3} \mathrm{Neu} 5 \mathrm{Ac}-$
nLc8-Cer species, with two different fatty acids in the ceramide.

\section{Acknowledgments}

Financial help of Hochschulbauförderungsgesetz, Land NordrheinWestfalen ( $\mathrm{HbfG})$ for the QTOF instrument, Interdisciplinary Centre for Clinical Research at University of Münster IZKF, Project C10 (JPK), and of the Deutsche Forschungsgemeinschaft DFG, SFB 549 Macromolecular Processing and Signaling in the Extracellular Matrix, Project B07 (JM), is gratefully acknowledged. The authors thank Željka Vukelić, University of Zagreb, Croatia, for providing the Cronassial standard and numerous additional information.

This work is part of WM's Ph.D. thesis in chemistry at the University of Münster.

\section{References}

1. Crocker, R.; Feizi, T. Curr. Opin. Struct. Biol. 1996, 6, 679-691.

2. Vestweber, D; Blanks, J. E. Physiol. Rev. 199, 79(1), 181.

3. Tiemeyer, M.; Swiedler, S. J.; Ishihara, M.; Moreland, M.; Schweingruber, H.; Hirtzer, P.; Brandley, B. K. Proc. Natl. Acad. Sci. USA 1991, 88, 1138-1142.

4. Stroud, M. R.; Handa, K.; Salyan, M. E. K.; Ito, K.; Levery, S. B.; Hakomori, S.; Reinhold, B. B.; Reinhold, V. Biochemistry 1996, $35,758-769$.

5. 20 Müthing, J.; Spanbroek, R.; Peter-Katalinić, J.; Hanisch, F. G.; Hanski, C.; Hasegawa, A.; Unland, F.; Lehmann, J.; Tschesche, H.; Egge, H. Glycobiology 1996, 6(2), 147.

6. Hakomori, S.; Handa, K.; Iwabuchi, K.; Yamamura, S.; Prinetti, A. Glycobiology 1998, 8(10), 11-19.

7. Müthing, J. Methods Enzymol. 2001, 382(2), 259-274.

8. Peter-Katalinić, J. Mass Spectrom. Rev. 1994, 13, 77-98.

9. Vukelić, Ž.; Metelmann, W.; Müthing, J.; Kos, M.; PeterKatalinić, J. Biol. Chem., in press.

10. De Jong, A. Mass Spectrom. Rev. 1999, 17(5), 311.

11. Shevchenko, A.; Chernushevich, I.; Ens, W.; Standing, K.G.; Thomson, B.; Wilm, M.; Mann, M. Rapid Commun. Mass Spectrom. 1997, 11, 1015.

12. Metelmann W.; Müthing, J.; Peter-Katalinić, J. Rapid Commun. Mass Spectrom. 2000, 14, 543-550.

13. Müthing, J.; Unland, F. J. Chromatogr. B. 1994, 658, 39-45.

14. Müthing, J.; Unland, F.; Heitmann, D.; Orlich, M.; Hanisch, F. G.; Peter-Katalinić, J.; Knäuper, V.; Tschesche, H.; Kelm, S.; Schauer, R.; Lehmann, J. Glycoconj. J. 1993, 10, 120-126.

15. Folch, J.; Lees, M.; Sloane, Stanley G. H. Biol. Chem. 1957, 226, 497-509.

16. Müthing, J.; Egge, H.; Kniep, B.; Mühlradt, P. F. Eur. J. Biochem. 1987, 163, 407-416. 
17. Ueno, K.; Ando, S.; Yu, R. K. J. Lipid Res. 1978, 19, 863871.

18. Svennerholm, L. J. Neurochem. 1956, 1, 42-53.

19. Verentchikov, A. N.; Ens, W.; Standing, K. G. Anal. Chem. 1994, 66, 126.

20. Alving, K.; Paulsen, H.; Peter-Katalinić, J. J. Mass Spectrom. 1999, 34, 395.

21. Domon, B.; Costello, C. E. Glycoconj. J. 1988, 5, 397-409.
22. IUPAC-IUB Commission on Biochemical Nomenclature (CNB). The nomenclature of lipids. Recommendations 1976. Eur. J. Biochem. 1977, 9, 11-21.

23. IUPAC-IUB Joint Commission on Biochemical Nomenclature (JCNB). Nomenclature of glycolipids. Recommendations 1997. Eur. J. Biochem. 1998, 257, 293-298.

24. Karas, M.; Bahr, U.; Dülcks, T.; Fresenius, J. Anal. Chem. 1999, $366,669-676$. 